\title{
Indirect mitotic nondisjunction in Vicia faba and Chinese hamster cells
}

\author{
Marco Rizzoni ${ }^{1}$, Caterina Tanzarella ${ }^{3}$, Bianca Gustavino ${ }^{1}$, Francesca Degrassi ${ }^{2}$, \\ Almerinda Guarino ${ }^{3}$, and Eleonora Vitagliano ${ }^{2}$ \\ ${ }^{1}$ Dipartimento di Biologia, Facolta' di Scienze M.F.N. II, Universita' di Roma "Torvergata", Via O. Raimondo, I-00173 Roma, Italy \\ ${ }^{2}$ Centro di Genetica Evoluzionistica del C.N.R. c/o and ${ }^{3}$ Dipartimento di Genetica e Biologia Molecolare, Facolta' di Scienze M.F.N. \\ Universita' di Roma "La Sapienza”, Piazzale A. Moro, I-00185 Roma, Italy
}

\begin{abstract}
The hypothesis of indirect mitotic nondisjunction was tested in plant and mammalian cells. This hypothesis states that micronuclei derived from lagging chromosomes or chromatids are able to perform DNA synthesis and undergo mitotic condensation synchronously with main nuclei. Hence, as chromosomes, they can be moved to spindle poles together with the chromosomes of the main nuclei during mitosis. In that way chromosomes "lost" as micronuclei can be reincorporated in the main nuclei. In order to test this, both Vicia faba meristematic cells and cells of a Chinese hamster line (Cl-1) were treated with low doses of colchicine. Mitotic anomalies, micronuclei and cells with a polyploid or aneuploid karyotype were scored at different fixation times. A detailed analysis was performed on single chromosome misdistributions, as well as on micronuclei and cells with aneuploid karyotypes derived from single chromosome misdistributions. Indirect mitotic nondisjunction was shown to play a primary role in the origin of aneuploid karyotypes in Vicia faba, but not in Cl-1 cells.
\end{abstract}

\section{Introduction}

In recent years several suggestions have been put forward to explain the mitotic misdistribution of single chromosomes (chromosome loss and nondisjunction). The time sequence of centromere separation has been suggested to be a reason for spontaneous mitotic nondisjunction (Vig 1984) based on studies with human lymphocytes (Vig 1981), Chinese hamster cells (Vig and Miltenburger 1976) and Vicia faba cells (Murata and Vig 1980). "Chromosome displacement" has been suggested to be the first step leading to chromosome loss and, to a lesser extent, to mitotic nondisjunction (Ford and Roberts 1983; Ford 1984, 1985). Another hypothesis is that prolonged exposure of kinetochores to cytoplasmic proteases, during mitotic arrest induced by spindle poisons, might lead to both nondisjunction and lagging chromosomes (Hsu et al. 1983; Hsu and Satya-Prakash 1985).

Lagging chromosomes and nondisjunction have been regarded as alternative phenomena, the former giving rise to chromosome loss, the latter to both chromosome loss and gain. We wanted to test whether lagging chromosomes or chromatids can lead to chromosome gain too. Lagging chromosomes and chromatids which are not included in

Offprint requests to: $\mathrm{M}$. Rizzoni daughter nuclei will give rise to micronuclei. If such micronuclei are able to perform DNA synthesis and mitotic condensation synchronously with the main nuclei, they can subsequently be integrated into main nuclei during the first mitosis following misdistribution. In fact, after disintegration of the nuclear envelopes, chromosomes from both micronucleus and main nucleus may move together to anaphase poles and form daughter nuclei with an additional chromosome. Such a process could be termed indirect mitotic nondisjunction, since chromosome gain would be produced besides chromosome loss (Fig. 1).

To test this hypothesis meristematic cells of secondary root tips of $V$. faba and cells from a stable Chinese hamster cell line (Cl-1) were treated with a dose of colchicine resulting in a good yield of single chromosome misdistribution but not in c-mitotic arrest of the whole cell population (Degrassi and Rizzoni 1982; Danford 1984). Using these doses, however, c-mitoses and multipolar mitoses are also induced. Mitotic anomalies, micronuclei, micronucleated and multinucleated cells in interphase and in mitosis, as well as polyploid and aneuploid cells, were scored to obtain a general picture of the induced damage.

To follow the process of indirect mitotic nondisjunction a detailed analysis was performed on single chromosome misdistribution and on its products at the subsequent mitosis. Thus lagging chromosomes and chromatids, prophase cells with single micronuclei and nearly diploid c-metaphase cells with a variant karyotype, i.e. monosomic cells, monosomic cells with a single micronucleus, diploid cells with a single micronucleus and trisomic cells, were scored (see Fig. 1). The DNA content of micronuclei derived from single laggards was measured in prophase cells of $V$. faba, and related to the ability of these micronuclei to undergo mitotic condensation synchronously with the main nuclei.

On the basis of the observed frequencies of single laggards (chromosomes or chromatids) and of single micronuclei in mitotic condensation in prophase cells, the expected frequencies of nearly diploid cells with a variant karyotype were calculated using simple equations (see Appendix). This allowed us to evaluate the role of indirect mitotic nondisjunction in the origin of aneuploidy.

\section{Materials and methods}

Materials and colchicine treatment. $V$. faba seeds, Weibullus akerböna, varj minor, with a standard karyotype $(2 n=12)$, were stored at $+4^{\circ} \mathrm{C}$ until use. The technique of Kihlman 


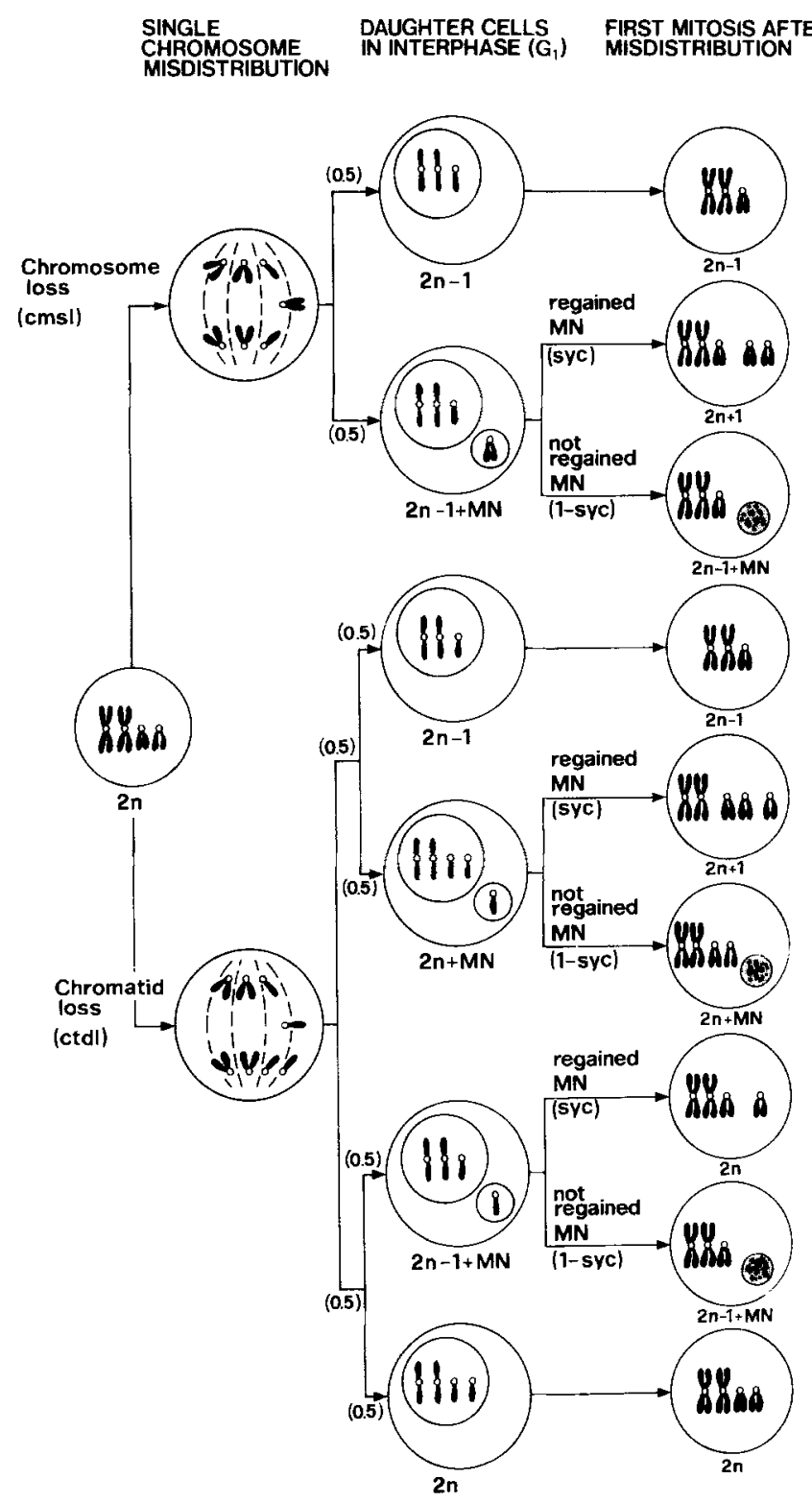

Fig. 1. Scheme for indirect mitotic nondisjunction by the reintegration into the main nucleus of a whole chromosome or chromatid "lost" in a micronucleus. The probabilities of the events involved in the production of variant karyotypes are given in parentheses. Micronuclei which are not mitotically condensed are represented as dotted circles at the first mitosis after misdistribution. $M N \mathrm{Mi}-$ cronucleus; cmsl probability of chromosome loss; ctdl probability of chromatid loss; syc probability of synchronous condensation of micronuclei with main nuclei

(1975) was used for germination. Plants were treated for $24 \mathrm{~h}$ with $3 \times 10^{-5} \mathrm{M}$ colchicine (Merck) when roots were $2-4 \mathrm{~cm}$ long, immersing roots in the colchicine solution. Colchicine was removed and the roots were washed. Treated root tips were fixed 24,44 and $64 \mathrm{~h}$ after the start of the treatment in a $3 / 1$ methanol/acetic acid mixture. Untreated roots were fixed at $24 \mathrm{~h}$. For each fixation time, $0.05 \%$ colchicine was added to half the samples from both control and treated roots $2 \mathrm{~h}$ before fixation to obtain cmetaphases. All root tips were Feulgen stained and squashed. The preparations were mounted in Canada balsam.
Cl-1 cells (modal chromosome number $s=21$ ) were cultivated on cover slips in Ham's F-10 medium supplemented with $10 \%$ foetal calf serum. Exponentially growing cells were treated with $10^{-7} \mathrm{M}$ colchicine (Merck) for $24 \mathrm{~h}$; colchicine was then removed and cells were washed with and reincubated in culture medium. The cells were fixed with a $3 / 1$ methanol/acetic acid mixture 24,48 , and $72 \mathrm{~h}$ after the beginning of the treatment. Untreated control cultures were fixed at $24 \mathrm{~h}$. For each fixation time $5 \times 10^{-5} \mathrm{M}$ colchicine was added to half the samples from both control and treated cultures $3 \mathrm{~h}$ before fixation to obtain c-metaphases. A $20 \mathrm{~min}$ hypotonic treatment with a $3 / 1 \mathrm{H}_{2} \mathrm{O} /$ medium mixture was carried out on these samples before fixation. Coverslips were stained with Giemsa and mounted in Canada balsam.

Scoring. Diameters of $2 / 3$ (Vicia faba) and 1/2 (Cl-1) of late telophase nuclei were chosen as borderline values to differentiate between micronuclei and small nuclei, taking into account the number and size of chromosomes of the two cell systems. Cells with two or more nuclei (with or without micronuclei) were classified as multinucleated cells. Cells with one or more micronuclei were classified as micronucleated cells. Micronuclei with a prophase appearance were classified as mitotically condensed.

Two thousand interphases were analysed for micronuclei, micronucleated and multinucleated cells, 200 prophases for micronuclei with or without mitotic condensation and for synchronous or asynchronous multinucleated cells, 200 metaphases for micronuclei, 200 anaphases for mitotic anomalies and micronuclei, and $200 \mathrm{c}$-metaphases for polyploid and aneuploid cells.

Single lagging chromosomes or chromatids were scored in 1000 ana/telophases $24 \mathrm{~h}$ after the beginning of treatments. Micronuclei with or without mitotic condensation in 50 single micronucleated prophase cells and different classes of nearly diploid cells with a variant karyotype in 1000 c-metaphases were scored $24 \mathrm{~h}(\mathrm{Cl}-1)$ and $44 \mathrm{~h}$ $(V . f a b a)$ after the start of treatment. These fixation times were chosen so as to analyse the cells in their second mitoses after the start of treatment. At these times the highest frequencies of tetraploid, hypotetraploid and micronucleated mitoses were observed, while octoploid and hypooctoploid mitoses were not found.

Metacentric (M) and subtelocentric (St) chromosomes can be easily distinguished in $V$. faba, its haploid karyotype being composed of five subtelocentric chromosomes of similar size and shape, and one large metacentric chromosome (see Kihlman 1975). Therefore we determined which type of chromosome was involved in misdistribution and in monosomy or trisomy.

$D N A$ content of micronuclei in prophase cells of $V$. faba. The DNA content of 50 single micronuclei in nearly diploid prophase cells of $V . f a b a$, fixed at 44 h, was measured with a Zeiss III Photomicroscope equipped with a 01 photometer. These micronuclei were classified as pycnotic (homogeneously stained, presumably dead or degenerating; see McLeish 1954), granular (showing a staining pattern similar to that of the main interphase nucleus; see McLeish 1954) or in mitotically condensed. The $4 C$ value (DNA content of a G2 nucleus) was determined by measuring $202 n$ prometaphases in the neighbourhood of the measured micronuclei. The standard error of the mean $4 C$ value was about 
$3 \%$. Repeated measurements of the same micronucleus showed a variation range of about $9 \%$ of the average value. Nearly diploid prophase main nuclei were accepted within a range between $(4 C-20 \%)$ and $(4 C+3 \%)$ to include both $2 n$ and $(2 n-1)$ nuclei. The upper limit for accepting micronuclei was $34 \%$ of $4 C$ (corresponding to two metacentric chromosomes). The limits for both main nuclei and micronuclei were chosen so that the products of single chromosome misdistribution only were studied. The DNA content of single chromosomes was measured in five $2 n$ c-metaphases.

\section{Results}

As expected after a treatment with a low dose of colchichine, c-mitoses, and multipolar and "disrupted" (see Ta-

Table 1. Frequencies of mitotic anomalies in ana/telophases of Vicia faba and $\mathrm{Cl}-1$ cells following a $24 \mathrm{~h}$ treatment with colchicine

\begin{tabular}{llll}
\hline Fixation & c-anaphases & $\begin{array}{l}\text { Multipolar/ } \\
\text { disrupted }^{\mathrm{a}} \\
\text { time (h) }\end{array}$ & $\begin{array}{l}\text { Single lagging } \\
\text { chromosomes/ } \\
\end{array}$ \\
& $(\%)$ & $(\%)$ & $(\%)$ \\
& & chromatids \\
\hline
\end{tabular}

\begin{tabular}{lccl}
\hline$V . f a b a$ & & & \\
Control & 0 & 0 & 0 \\
24 & 13.5 & 9.5 & 6 \\
44 & 7 & 15.5 & 7 \\
64 & 4.5 & 8 & 3.5 \\
Cl-1 cells & & & \\
Control & 0 & 4 & 0 \\
24 & 2 & 10 & 7 \\
48 & 3 & 5 & 2 \\
72 & 1 & 14 & 6 \\
\hline
\end{tabular}

Each sample consisted of 200 cells. Colchicine concentration was $3 \times 10^{-5} \mathrm{M}$ for $V$. faba and $10^{-7} \mathrm{M}$ for $\mathrm{Cl}-1$ cells

a "Disrupted" ana/telophases, those with multiple laggards, with or without multipolar spindles ble 1 for definition) mitoses were observed in both cell types together with single lagging chromosomes or chromatids (Table 1) (Östergren and Levan 1943; Deysson 1968; Gustavino et al. 1987). Multinucleated and multiply micronucleated cells, derived from c-mitoses and multipolar/disrupted mitoses, were observed together with singly micronucleated cells, which arise mainly from single lagging chromosomes or chromatids (Stubblefield 1964; Kato and Sandberg 1967) (Table 2). Polyploid cells, derived from cmitoses, and multiply aneuploid cells, derived from multipolar/disrupted mitoses, (Deysson 1968; Hsu et al. 1983; Satya-Prakash et al. 1984) were observed together with monosomic and trisomic cells, resulting from single chromosome misdistribution (Table 3).

The ratio between the frequencies of single lagging chromosomes or chromatids in ana/telophases (Table 1) and of singly micronucleated cells in interphases (Table 2) is close to $2: 1$, indicating that most cells passed a mitosis and that each single laggard gave rise to a single micronucleus in one of the daughter cells.

The frequency of mitotic anomalies decreased with time, yet it never reached zero. Mitotically condensed micronuclei were only observed in prophase cells (Table 2, Fig. 2b, h). Neither premature chromosome condensation (PCC) (Stubblefield 1964; Kato and Sandberg 1967; Obe and Beek 1982 ) in mitotic cells nor delayed mitotic condensation in metaphase, anaphase, telophase and interphase cells was observed for micronuclei in either cell system.

Single lagging chromatids were more frequent than single lagging chromosomes in both $V$. faba (ratio close to 6:1) and Cl-1 cells (close to 4:3) (Table 4, Fig. 2a, g). On the other hand, only lagging chromosomes were found in Don cells treated with diazepam (Hsu et al. 1983). A ratio close to 5:1 was found between subtelocentric and metacentric lagging chromosomes or chromatids in $V$. faba (Table 4). This ratio is expected assuming a random involvement of each chromosome in misdistribution.

The frequency of single micronuclei in synchronous mitotic condensation with main nuclei in prophase was $30 \%$

Table 2. Frequencies of micronuclei, and micronucleate and multinucleate cells of Vicia faba and Cl-1 following a 24 h-treatment with colchicine $^{a}$

\begin{tabular}{|c|c|c|c|c|c|c|c|c|}
\hline \multirow{2}{*}{$\begin{array}{l}\text { Fixation } \\
\text { time (h) }\end{array}$} & \multicolumn{4}{|l|}{ Interphases ${ }^{b}$} & \multicolumn{2}{|l|}{ Prophases $^{b}$} & \multirow{2}{*}{$\begin{array}{l}\text { Metaphases } \\
\text { Micronuclei } \\
(\%)\end{array}$} & \multirow{2}{*}{$\begin{array}{l}\text { Ana/ } \\
\text { telophases } \\
\text { Micronuclei } \\
(\%)\end{array}$} \\
\hline & $\begin{array}{l}\text { Singly } \\
\text { micronucleated } \\
\text { cells } \\
(\%)\end{array}$ & $\begin{array}{l}\text { Multiply } \\
\text { micronucleated } \\
\text { cells } \\
(\%)\end{array}$ & $\begin{array}{l}\text { Total } \\
\text { micronuclei } \\
(\%)\end{array}$ & $\begin{array}{l}\text { Multinucleated } \\
\text { cells } \\
(\%)\end{array}$ & $\begin{array}{l}\text { Micronuclei } \\
(\%)\end{array}$ & $\begin{array}{l}\text { Multinucleated } \\
\text { cells } \\
(\%)\end{array}$ & & \\
\hline \multicolumn{9}{|l|}{$V \cdot f a b a$} \\
\hline $\begin{array}{l}\text { Control } \\
24 \\
44 \\
64\end{array}$ & $\begin{array}{l}0.05 \\
1.65 \\
2.7 \\
3.25\end{array}$ & $\begin{array}{l}0 \\
0.05 \\
0.25 \\
0.3\end{array}$ & $\begin{array}{l}0.05 \\
1.9 \\
3.95 \\
4.3\end{array}$ & $\begin{array}{l}0 \\
0.55 \\
1.4 \\
1.65\end{array}$ & $\begin{array}{l}0 \\
0.5(0)^{\mathrm{c}} \\
3.5(1) \\
4.5(1.5)\end{array}$ & $\begin{array}{l}0 \\
0 \\
3.5(1.5)^{d} \\
5 \quad(3.5)\end{array}$ & $\begin{array}{l}0 \\
0 \\
3(0)^{\mathrm{c}} \\
4(0)\end{array}$ & $\begin{array}{l}0 \\
0 \\
3 \quad(0)^{\mathrm{c}} \\
4.5(0)\end{array}$ \\
\hline \multicolumn{9}{|l|}{ Cl-1 cells } \\
\hline $\begin{array}{l}\text { Control } \\
24 \\
48 \\
72\end{array}$ & $\begin{array}{l}0.5 \\
1.7 \\
3.2 \\
1.8\end{array}$ & $\begin{array}{l}0 \\
0.3 \\
0.4 \\
0\end{array}$ & $\begin{array}{l}0.5 \\
2.45 \\
4.15 \\
1.8\end{array}$ & $\begin{array}{l}0.3 \\
0.3 \\
1.05 \\
0\end{array}$ & $\begin{array}{ll}0 & \\
5 & (4) \\
2 & (1) \\
5 & (5)\end{array}$ & $\begin{array}{ll}0 & \\
0 & \\
2 & (2) \\
0 & \end{array}$ & $\begin{array}{l}0 \\
1(0) \\
2(0) \\
1(0)\end{array}$ & $\begin{array}{l}0.5(0) \\
0 \\
0 \\
0\end{array}$ \\
\hline
\end{tabular}

a Concentrations of colchicine as in Table 1

b Numbers analysed were 2000 for interphase and 200 each for prophase, metaphase and ana/telophase cells

c Numbers in parentheses represent percentages of mitotically condensed micronuclei

d Numbers in parentheses represent percentages of synchronous multinucleated cells 
Table 3. Frequencies of polyploid and aneuploid cells following a $24 \mathrm{~h}$ treatment of Vicia faba and Cl-1 cells with colchicine ${ }^{\mathrm{a}}$

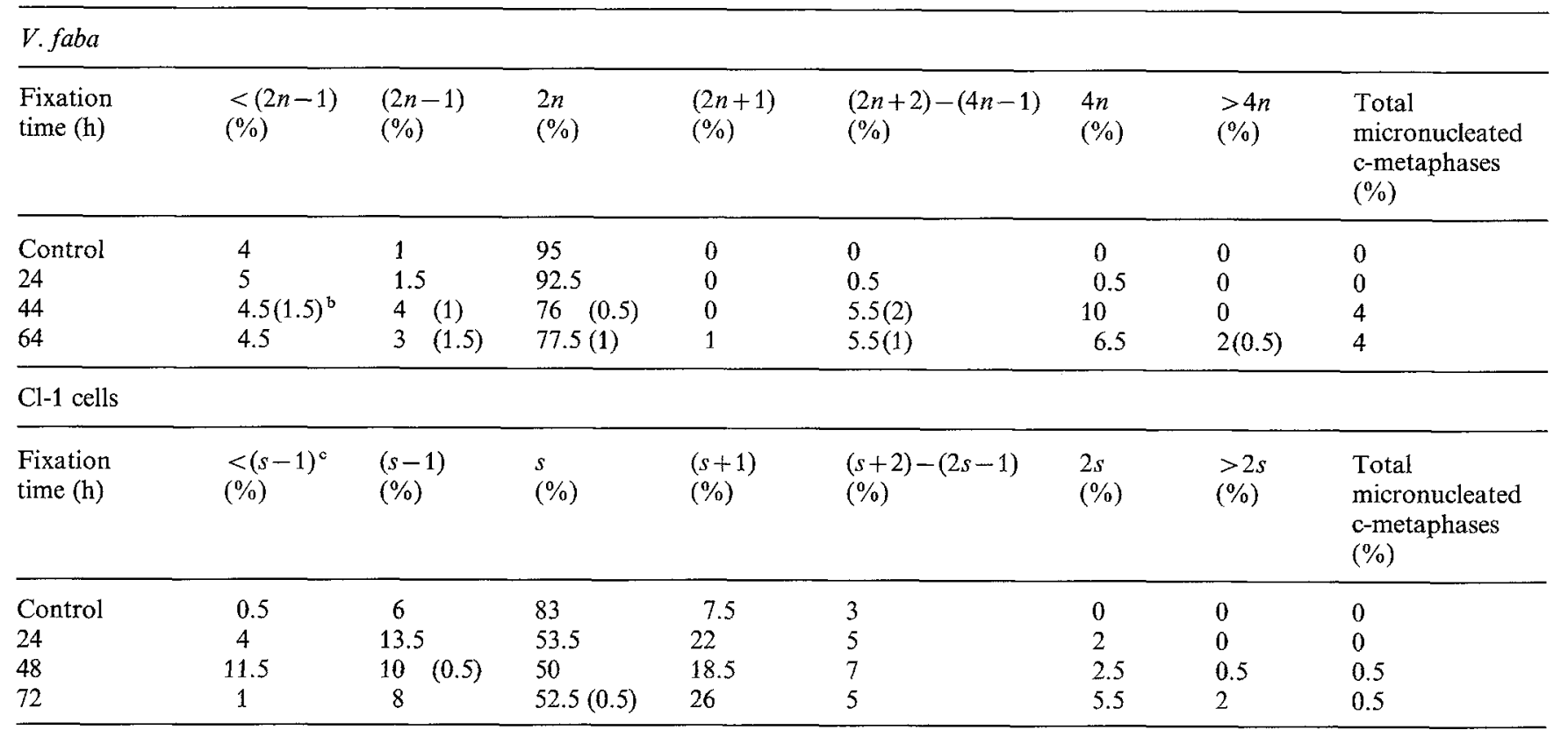

Each sample consisted of 200 c-metaphases

a Colchicine concentrations as in Table 1

${ }^{b}$ Numbers in parentheses represent percentages of micronuclei not mitotically condensed

c $s$ Modal chromosome number of $\mathrm{Cl}-1$ cells

Table 4. Analysis of the frequencies of single lagging chromosomes/chromatids and of their products at the first mitosis after misdistribution in colchicine-treated Vicia faba and $\mathrm{Cl}-1$ cells

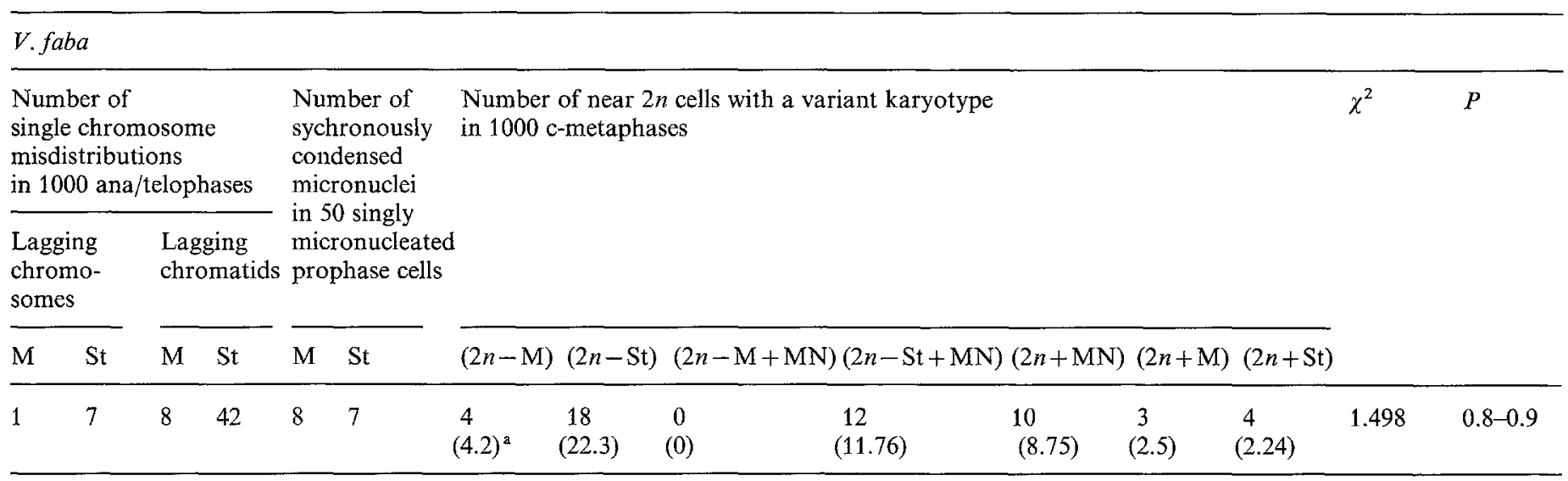

Cl-1 cells

\begin{tabular}{|c|c|c|c|c|c|c|c|}
\hline \multicolumn{2}{|c|}{$\begin{array}{l}\text { Number of } \\
\text { single chromosome } \\
\text { misdistributions } \\
\text { in } 1000 \text { ana/telophases }\end{array}$} & \multirow{2}{*}{$\begin{array}{l}\text { Number of } \\
\text { sychronously } \\
\text { condensed } \\
\text { micronuclei } \\
\text { in } 50 \text { singly } \\
\text { micronucleated } \\
\text { prophase cells }\end{array}$} & \multicolumn{3}{|c|}{$\begin{array}{l}\text { Number of near } s \text { cells with a variant karyotype } \\
\text { in } 1000 \mathrm{c} \text {-metaphases }\end{array}$} & \multirow[t]{2}{*}{$\chi^{2}$} & \multirow[t]{2}{*}{$P$} \\
\hline $\begin{array}{l}\text { Lagging } \\
\text { chromo- } \\
\text { somes }\end{array}$ & $\begin{array}{l}\text { Lagging } \\
\text { chromatids }\end{array}$ & & $(s-1)$ & $(s-1+\mathrm{MN}) /(s+\mathrm{MN})$ & $(s+1)$ & & \\
\hline 29 & 40 & 34 & $\begin{array}{l}141 \\
(84.5)\end{array}$ & $\begin{array}{c}4 \\
(11)\end{array}$ & $\begin{array}{l}204 \\
(91.6)\end{array}$ & 212.38 & $<0.001$ \\
\hline
\end{tabular}

$M$ Metacentric; St subtelocentric; $M N$ micronucleus; $s$ modal chromosome number in Cl-1 cells. Colchicine concentrations as in Table 1. Lagging chromosomes/chromatids were scored at $24 \mathrm{~h}$ after the beginning of the treatment for both systems; micronuclei in prophase cells and near $2 n / s$ cells with a variant karyotype were scored at $44 \mathrm{~h}$ in Vicia faba and at $24 \mathrm{~h}$ in Cl-1 (see Materials and methods). The values for synchronously condensed micronuclei in prophase cells were taken from Figure 3 for $V$. faba and were classified as containing metacentric or subtelocentric chromosomes on the basis of DNA amount (see Fig. 3). Chi square values were calculated by pooling the classes with low expected frequencies and computing together the other ploidy classes not described in this table.

${ }^{a}$ The expected frequencies of the different classes of near $2 \mathrm{n} / \mathrm{s}$ cells with a variant karyotype are given in parentheses and calculated on the basis of the equations described in Appendix 

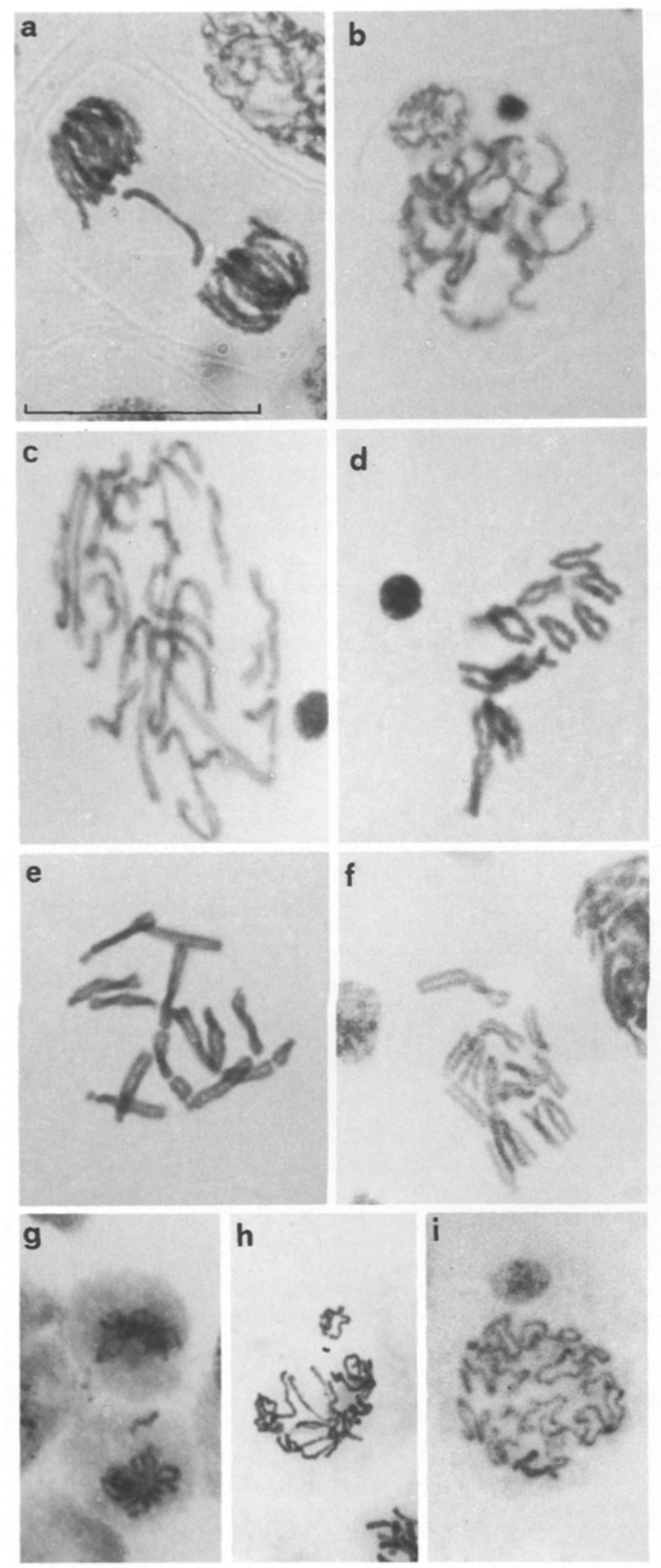

Fig. 2a-f. Meristematic cells of Vicia faba root tips. a Single lagging metacentric chromatid in a telophase cell. b Mitotically condensed micronucleus in a prophase cell. c A non-mitotically condensed micronucleus in a prophase cell. d A $2 n+$ micronucleus c-metaphase. e A $2 n-1$ c-metaphase lacking a subtelocentric chromosome. f A $2 n+1$ c-metaphase with an extra metacentric chromosome. $g-\mathbf{i}$ Cells from the stable Chinese hamster line $\mathrm{Cl}-1$. g A lagging chromatid in an ana/telophase cell. h A synchronously micronucleus in a prophase cell. i A non-mitotically condensed micronucleus in a prophase cell. Bar represents $10 \mu \mathrm{m}$
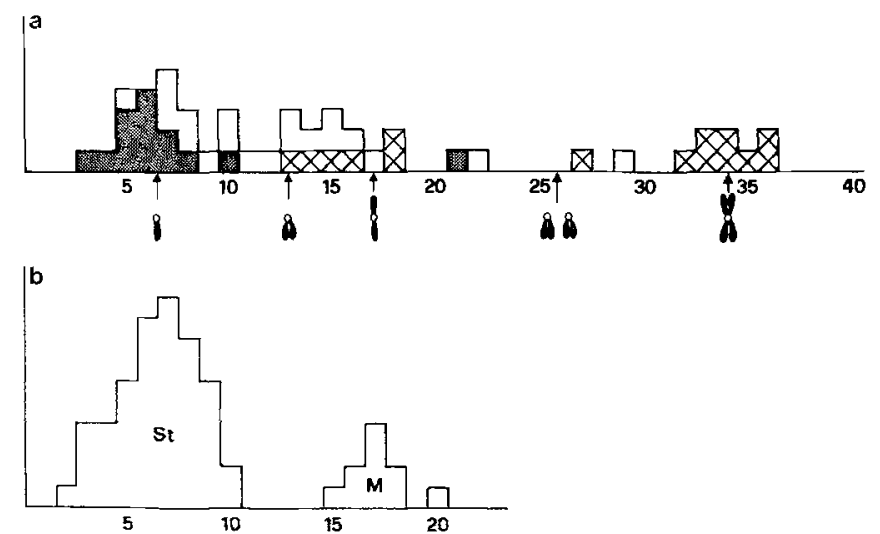

Fig. 3. a DNA content of single micronuclei of 50 nearly diploid prophase cells of Vicia faba root tips fixed $44 \mathrm{~h}$ after the beginning of a $24 \mathrm{~h}$ treatment with $3 \times 10^{-5} \mathrm{M}$ colchicine. Abscissa, DNA content expressed as a percentage of $2 c$ (the value of a Gl nucleus). Ordinate, Number of micronuclei. Grey areas, pycnotic micronuclei; white areas, granular micronuclei; cross-hatched areas, micronuclei synchronously condensed with main nuclei. b DNA content of single chromosomes measured in $52 n$ c-metaphases, expressed as percentage of the DNA amount of the whole c-metaphase (4c). Abscissa, DNA content of single subtelocentric (St) and metacentric $(M)$ chromosomes. Ordinate, Number of chronosomes

in $V . f a b a$ and $68 \%$ in $\mathrm{Cl}-1$ cells (Table 4). In nearly diploid prophase cells of $V$. faba, pycnotic micronuclei had a DNA content corresponding to unreplicated chromatids, and micronuclei in synchronous mitotic condensation with main nuclei had a DNA content corresponding to replicated chromosomes. The DNA content of granular micronuclei had an intermediate value.

On the basis of DNA content, a ratio close to $1: 1$ was found between micronuclei in synchronous mitotic condensation containing a subtelocentric or a metacentric chromosome (Fig. 3). Comparing this ratio with that found for laggards $(5: 1)$ it can be assumed that micronuclei containing metacentric chromosomes undergo synchronous mitotic condensation about five times more often than micronuclei containing subtelocentric chromosomes. The relative frequency of micronuclei in synchronous mitotic condensation containing a metacentric chromosome among all micronuclei in prophase cells $(8 / 50)$ is very close to that of metacentric laggards among all laggards (9/58) (Table 4). This suggests that all metacentric chromosomes in micronuclei undergo synchronous mitotic condensation with main nuclei.

On the basis of the frequencies of lagging chromosomes or chromatids and micronuclei in synchronous mitotic condensation with main nuclei, we calculated the frequencies of nearly diploid cells with a variant karyotype expected to occur at the first mitosis following misdistribution (Table 4) (see Fig. 1; Appendix).

Observed and expected frequencies were very similar in $V$. $f a b a$ cells. A ratio close to 5:1 was found between $(2 n-1)$ cells lacking a subtelocentric chromosome (Fig. 2e) and those lacking a metacentric one. A ratio close to $1: 1$ was found between $(2 n+1)$ cells with an extra subtelocentric chromosome and those with an extra metacentric one (Fig. $2 \mathrm{f})$. No $(2 n-1+\mathrm{MN})$ cell was found lacking a metacentric chromosome. 
The observed frequencies of $(s-1)$ and especially $(s+1)$ cells in $\mathrm{Cl}-1$ were much higher than those expected (Table 4; see also Danford 1984).

\section{Discussion}

Our data strongly suggest that lagging chromosomes or chromatids and indirect mitotic nondisjunction play a primary role in giving rise to aneuploid karyotypes following single chromosome misdistribution in $V$. $f a b a$, while direct nondisjunction is a rare event. Moreover, while nearly all metacentric chromosomes are regained by main nuclei, about one-sixth of subtelocentric ones are regained.

In contrast direct nondisjunction is likely to play a major role in inducing aneuploid karyotypes in $\mathrm{Cl}-1$ cells. The real occurrence of indirect mitotic nondisjunction cannot be verified in this cell type, due to the high incidence of direct nondisjunction.

To account for the difference observed between $V$. faba and $\mathrm{Cl}-1$ cells consideration could be made of the forces acting in distribution or misdistribution of chromosomes during mitosis (forces moving kinetochores towards the poles, the association between sister centromeres) in plant and animal cells. Particular attention should be given to the fate of micronuclei. Reports in the literature on replication and mitotic condensation of micronuclei have mainly been concerned with micronuclei derived from acentric fragments: in cultured mammalian cells an asynchronous pattern of DNA synthesis has been shown, (Kato and Sandberg 1968; Ikeuchi 1973; Griffiths and Carpenter 1979) which has been linked to PCC. In human lymphocytes both asynchronous (Obe and Beek 1975) and synchronous (Pincu et al. 1985) DNA synthesis pattern have been found. In $V$. faba DNA replication of micronuclei has been shown to occur either synchronously with main nuclei or not at all (Das 1962; Scott and Evans 1964). In this cell system micronuclei were found to undergo "normal" mitotic condensation or to remain without mitotic condensation in an interphase state (McLeish 1954; Scott and Evans 1964). Also, PCC in G2 of micronuclei containing whole chromosomes has been found in mammalian cells (Obe and Beek 1975; Raman 1980; for review see Obe and Beek 1982). However, a high degree of synchrony has been found among micronuclei both in DNA synthesis and in mitosis in PtK-1 cells (Ghosh and Paweletz 1984). In summary micronuclear PCC is rather common in mammalian cells, while it is very rare in plant cells (see Obe and Beek 1982).

Our data in $V$. faba show that micronuclei containing single chromosomes are derived mostly from chromatids; some micronuclei replicate before cells enter mitosis and undergo mitotic condensation synchronously with main nuclei. In contrast, micronuclei which have not completed DNA synthesis cannot undergo mitotic condensation.

Some aspects of the process of indirect mitotic nondisjunction should be mentioned:

1. Indirect mitotic nondisjunction gives rise to both $(2 n+1)$ and $(2 n-1)$ cells following single lagging chromosomes or chromatids, although $(2 n+1)$ cells are less frequent because not all micronuclei are regained.

2. Micronuclei with more than one chromosome or nuclei belonging to multinucleated cells could be regained following the same pathway as described for single chromosome misdistribution, but this cannot be demonstrated unambiguously. Indirect evidence for this is provided by the high degree of synchrony in multinucleated prophase cells (Table 2). Thus, multiple aneuploidies can appear at the first mitosis after misdistribution by synchronous mitotic condensation of different nuclei and/or by asynchrony and loss (perhaps through PCC; see Stubblefield 1964; Kato and Sandberg 1967) of uncondensed nuclei (Rainaldi et al. 1987). Recently, selective chromosome replication has been suggested to be the reason for the appearance of hypotetraploid aneuploids from multinucleated cells following cmitosis (Frackowiak et al. 1986).

3. Different chromosomes in micronuclei might have different probabilities of being regained by main nuclei. Our data show that in $V$. faba this probability is close to 1 for the metacentric chromosome and to $1 / 6$ on average, for the subtelocentric chromosomes. A role of nucleolar organizer regions (NORs) within micronuclei in allowing synchronous mitotic condensation might explain this result, as NORs are located on the metacentric chromosome of V. faba (McLeish 1954; Das 1962; Scott and Evans 1964). 4. In the absence of micronuclei, aneuploid karyotypes are selected according to their genetic imbalance. During the time between micronucleus formation and its elimination or integration, selection on the "divided karyotype" may take place at the cellular level, as one chromosome is in the micronucleus and the others are in the main nucleus. It can be asked whether there is a functional integration between the micronucleus and main nucleus, i.e. whether a $(2 n-1+\mathrm{MN})$ cell acts as a $(2 n-1)$ cell or as a $(2 n) /(2 n+$ 1) cell. At the same time, selection on the micronucleus chromosome also takes place (see point 3 ). Divided karyotype and micronucleus chromosome selections act together giving rise to the starting frequencies of integrated aneuploid karyotypes. Non-random involvement of chromosomes in aneuploidy, which has been explained by the time sequence of centromere separation (Vig and Miltenburger 1976; Murata and Vig 1980; Vig 1984) or by differential chromosome displacement (Ford and Lester 1982; Ford 1984,1985 ) could also be explained by the selective process described here.

Acknowledgements. Thanks are due to Prof. F. Palitti, Dr. F. Spirito and Dr. P. Perticone for critically reading the manuscript. We are very grateful to Prof. G. Obe for valuable help in reviewing our work. We also wish to acknowledge the Shenker Foundation for a fellowship award to one of us (B.G.) This work was supported by Progetto Strategico "Mutagenesi" C.N.R. and Progetto di Interesse Nazionale "Processi di Mutazione Genetica" M.P.I.

\section{Appendix}

Calculation of the expected frequencies of cells with variant karyotypes after indirect mitotic nondisjunction

The expected relative frequencies of nearly $2 n / \mathrm{s}$ cells with a variant karyotype at the first mitosis after misdistribution can be easily calculated on the basis of the hypothesis delineated in Figure 1. Equations for each class of cells with a variant karyotype are derived by multiplying the probabilities of single events leading to that class of cells through a pathway and, if more than one pathway, by adding together the different probabilities of pathways leading to the same class of cells. The following assumptions were made:

1. No direct nondisjunction occurred. 
2. Each class of single laggards had the same probability close to 1 - to result in single micronuclei.

3. Each lagging chromatid had the same probability $(0.5)$ of being incorporated as a micronucleus either into the diploid or in the monosomic daughter cell.

4. Each class of nearly $2 n / \mathrm{s}$ cells with a variant karyotype had the same probability of reaching the first mitosis after single chromosome misdistribution as the diploid/modal ones.

5. Only micronuclei which had performed chromosome replication could undergo mitotic condensation synchronously with main nuclei.

6. Each chromosome had its own probability of undergoing mitotic condensation, as a micronucleus, synchronously with they main nucleus, regardless of whether it was lost as a chromosome or a chromatid.

The following symbols are used in the equations:

$M N$ Micronucleus;

cmsl probability of chromosome loss, measured as the relative frequency of ana/telophase with a single lagging chromosome among all the ana/telophases;

ctdl probability of chromatid loss, measured as the relative frequency of ana/telophases with a single lagging chromatid among all the ana/telophases;

syc probability of synchronous condensation of micronuclei with main nuclei, measured as the relative frequency of single micronuclei synchronously condensed with main nuclei in prophase cells, among all single micronuclei in prophase cells at the first mitosis after misdistribution;

$f$ expected relatively frequency of cells with the variant karyotype (in parentheses) among all the c-metaphases at the first mitosis after misdistribution;

contr relative frequency of cells with a given variant karyotype among all the c-metaphases in untreated cell populations. cells:

Thus, the following equations can be written for $\mathrm{Cl}-1$

$f(s-1)=0.5 \mathrm{cmsl}+0.25 \mathrm{ctdl}+$ contr

$f(s-1+\mathrm{MN})=(1-\mathrm{syc})(0.5 \mathrm{cmsl}+0.25 \mathrm{ctdl})+\mathrm{contr}$

$f(s+\mathrm{MN})=(1-\mathrm{syc}) 0.25 \mathrm{ctdl}+\mathrm{contr}$

$f(s+1)=\operatorname{syc}(0.5 \mathrm{cmsl}+0.25 \mathrm{ctdl})+$ contr.

Cells with a variant karyotype derived from single chromosome/chromatid misdistributions in cells with a chromosome number different from $s$ were neglected in the equations, due to their low frequencies in control cultures. For $V$. faba cells split equations can be written for metacentric (M) and subtelocentric (St) chromosomes (see Materials and methods) as follows:

$f(2 n-\mathrm{M})=0.5 \mathrm{Mcmsl}+0.25 \mathrm{Mctdl}+\mathrm{contr}$

$f(2 n-\mathrm{St})=0.5 \mathrm{Stcms} 1+0.25 \mathrm{Stctd} \mathrm{l}+\mathrm{contr}$

$f(2 n-\mathrm{M}+\mathrm{MN})=(1-\mathrm{Msyc} *)(0.5 \mathrm{Mcmsl}+0.25 \mathrm{Mctdl})+$ contr

$f(2 n-\mathrm{St}+\mathrm{MN})=\left(1-\mathrm{Stsyc}^{*}\right)(0.5 \mathrm{Stcmsl}+0.25 \mathrm{Stctdl})+$ contr

$f(2 n+\mathrm{MN})=(1-\mathrm{syc}) 0.25 \mathrm{ctdl}+\mathrm{contr}$

$f(2 n+\mathrm{M})=\mathrm{Msyc}^{*}(0.5 \mathrm{Mcmsl}+0.25 \mathrm{Mctdl})+\mathrm{contr}$

$f(2 n+\mathrm{ST})=$ Stsyc $*(0.5 \mathrm{Stcmsl}+0.25 \mathrm{Stctdl})+$ contr; where syc $=$ Msyc + Stsyc.

Msyc, Msyc*, Stsyc and Stsyc* are the relative frequencies of single micronuclei containing a metacentric or a subtelo- centric chromosome, which showed synchronous mitotic condensation with main nuclei. Msyc* and Stsyc* were calculated on the populations of single micronuclei containing a metacentric or a subtelocentric chromosome respectively, as opposed to Msyc and Stsyc, which were calculated on the whole micronucleus population. A ratio close to $5: 1$ found between subtelocentric and metacentric laggards (Table 4; see text) makes us to assume that the same ratio occurs between micronuclei containing subtelocentric and metacentric chromosomes. The following relationship can therefore be assumed:

Msyc $=(1 / 6)$ Msyc $^{*} ;$ Stsyc $=(5 / 6)$ Stsyc* .

We have also assumed that Msyc* $=1$ on the basis of the data (see Results), that is all micronuclei containing a metacentric chromosome undergo mitotic condensation in synchrony with main nuclei. On this basis it can be easily verified that: Stsyc* $=(6 / 5)(\mathrm{syc}-1 / 6)=0.16$.

The expected frequencies of the nearly $2 n / s$ cells with a variant karyotype were calculated giving the following values to the variables of the equations, on the basis of the empirical data (see Table 4):

For $\mathrm{Cl}-1$ cells: $\mathrm{cmsl}=0.029$; $\mathrm{ctdl}=0.04 ; \mathrm{syc}=0.68 ;(s-$ 1) $\operatorname{contr}=0.06 ;(s-1+\mathrm{MN}) \operatorname{contr}=0 ;(s+\mathrm{MN}) \operatorname{contr}=0$; $(s+1)$ contr $=0.075$.

For V. faba cells: $\mathrm{Stcmsl}=0.007 ; \mathrm{Mcmsl}=0.001 ; \mathrm{Stctdl}=$ $0.042 ; \mathrm{Mctdl}=0.008 ; \mathrm{Stsyc}^{*}=0.16 ; \mathrm{Msyc}^{*}=1 ; \mathrm{syc}=0.3$; $(2 n-\mathrm{M})$ contr $=0.0017 ;(2 n-\mathrm{St}) \mathrm{contr}=0.0083 ;(2 n-\mathrm{M}+$ $\mathrm{MN})$ contr $=0 ; \quad(2 n-\mathrm{St}+\mathrm{MN}) \mathrm{contr}=0 ; \quad(2 n+$ $\mathrm{MN}) \operatorname{contr}=0 ;(2 n+\mathrm{M}) \operatorname{contr}=0 ;(2 n+\mathrm{St}) \operatorname{contr}=0$.

\section{References}

Danford N (1984) Measurement of levels of aneuploidy in mammalian cells using a modified hypotonic treatment. Mutat Res 139:127-132

Das NK (1962) Synthetic capacities of chromosome fragments correlated with their ability to maintain nucleolar material. J Cell Biol 15:121-130

Degrassi F, Rizzoni M (1982) Micronucleus test in Vicia faba root tips to detect mutagen damage in fresh-water pollution. Mutat Res 97:19-33

Deysson B (1968) Antimitotic substances. Int Rev Cytol 24:99-148

Ford JH (1984) Spindle microtubular dysfunction in mothers of Down Syndrome children. Hum Genet 68:295-298

Ford JH (1985) A model for the mechanism of aneuploidy involving chromosome displacement. In: Dellarco VL, Voytek PE, Hollaender A (eds) Aneuploidy, ethiology and mechanisms. Plenum Press, NY, pp 291-295

Ford JH, Lester P (1982) Factors affecting the displacement of human chromosomes from the metaphase plate. Cytogenet Cell Genet 33:327-332

Ford JH, Roberts CG (1983) Displacement of chromosomes in mitosis: a technique for assessing differential chromosome error. Cytogenet Cell Genet 36:537-541

Frackowiak S, Labidi B, Hernandez-Verdun D, Bouteille M (1986) Preservation of chromosome integrity during micronucleation induced by colchicine in PtK-1 cells. Chromosoma 94:468-474

Ghosh S, Paweletz N (1984) Synchronous DNA synthesis and mitosis in multinucleate cells with one chromosome in each nucleus. Chromosoma 89:197-200

Griffiths TD, Carpenter JG (1979) Premature chromosome condensation following $X$-irradiation of mammalian cells: expression time and dose response. Radiat Res 79:187-202

Gustavino B, Vitagliano E, Scottili A, Rizzoni M (1987) A comparison between short term evolution of micronuclei induced by 
$\mathrm{X}$ rays and colchicine in root tips of Vivia faba. Mutat Res 192:109-121

Hsu TC, Satya-Prakash KL (1985) Aneuploidy induction by mitotic arrestants in animal cell systems: possible mechanisms. In: Dellarco VL, Voytek PE, Hollaender A (eds) Aneuploidy, ethiology and mechanisms. Plenum Press, NY, pp 279-289

Hsu TC, Liang JL, Shirley LR (1983) Aneuploidy induction by mitotic arrestants: effects of diazepam on diploid Chinese hamster cells. Mutat Res 122:201-209

Ikeuchi T (1973) Chromosome pulverization in Chinese hamster multinucleate cells induced by bleomycin and cytochalasin-B. Jpn J Genet 48:391-405

Kato H, Sandberg AA (1967) Chromosome pulverization in human binucleate cells following colcemide treatment. J Cell Biol $34: 35-45$

Kato H, Sandberg AA (1968) Chromosome pulverization in human cells with micronuclei. J Natl Cancer Inst 40:167-179

Kihlman BA (1975) Root tips of Vicia faba for the study of the induction of chromosomal aberrations. Mutat Res 31:401-412

McLeish J (1954) The consequences of localized chromosome breakage. Heredity $8: 385-407$

Murata M, Vig BK (1980) Sequence of centromere separation: analysis of mitotic chromosomes in a reconstructed karyotype of Vicia faba. L Biol Zentralbl 99:686-693

Obe G, Beek B (1975) The human leukocyte test system. VII. Further investigations concerning micronucleus-derived premature chromosome condensation. Humangenetik 30:143-154

Obe G, Beek B (1982) Premature chromosome condensation in micronuclei. In: Rao PT, Johnson RT, Sperling K (eds) Premature chromosome condensation. Application in basic, clinical and mutation research. Academic Press, NY, pp 113-130
Östergren G, Levan A (1943) The connection between c-mitotic activity and water solubility in some monocyclic compounds. Hereditas 29:496-498

Pincu M, Callisen H, Norman A (1985) DNA content of micronuclei in human lymphocytes. Int J Radiat Biol 47:423-432

Rainaldi G, Flori L, Colella CL, Mariani T, Piras A, Simi S, Simili M (1987) Analysis by BrudR-labelling technique of induced aneuploidy in mammalian cells in culture. Mutat Res $177: 255-260$

Raman R (1980) Asynchrony in proliferation of sister nuclei in colcemid-induced polykaryons of muntjak lymphocytes. Indian J Exp Biol 18:115-119

Satya-Prakash KL, Hsu TC, Wheeler WJ (1984) Metaphase arrest, anaphase recovery and aneuploidy induction in cultured Chinese hamster cells following exposure to mitotic arrestants. Anticancer Res 4:351-356

Scott D, Evans HJ (1964) Influence of the nucleolus on DNA synthesis and mitosis in Vicia faba. Exp Cell Res 36:145-159

Stubblefield E (1964) DNA synthesis and chromosomal morphology of Chinese hamster cells in media containing N-deacetyl-Nmethylcolchcine (Colcemid). Symp Int Soc Cell Biol. 3:223-248

Vig BK (1981) Sequence of centromere separation: analysis of mitotic chromosomes in man. Hum Genet 57:247-252

Vig BK (1984) Sequence of centromere separation: another mechanism for the origin of non disjunction. Hum Genet 66:239-243

Vig BK, Miltenburger MG (1976) Sequence of centromere separation of mitotic chromosomes in Chinese hamster. Chromosoma $5: 75-80$

Received December 10, 1986/in revised form October 19, 1988 Accepted by T.C. Hsu 